\title{
Prevenção da Doença Renal Crônica entre Adolescentes: um Relato de Experiência
}

Prevention of Chronic Kidney Disease among Adolescents: an Experience Report

\author{
Geisiane de Souza Santos ${ }^{1}$ \\ Valquíria Carvalho Silva ${ }^{2}$ \\ Wbiratan de Lima Souza ${ }^{3}$ \\ Lucimere Maria dos Santos ${ }^{4}$
}

\begin{abstract}
${ }^{1}$ Enfermeira. Mestre pelo Programa de Pós-Graduação Mestrado Profissional Enfermagem Assistencial (MPEA) da Escola de Enfermagem Aurora de Afonso Costa, da Universidade Federal Fluminense (EEAAC/UFF). Especialista em Nefrologia. Saquarema. Rio de Janeiro - Brasil. E-mail: geisisantos10@gmail.com

${ }^{2}$ Enfermeira. Mestre pelo Programa de Pós-Graduação Mestrado Profissional Enfermagem Assistencial (MPEA) da Escola de Enfermagem Aurora de Afonso Costa, da Universidade Federal Fluminense (EEAAC/UFF). Especialista em Saúde do Idoso pelo Programa de Residência Multiprofissional em Saúde do Hospital Universitário Antônio Pedro (HUAP/UFF). Rio Bonito. Rio de Janeiro - Brasil. Email:valquiria carvalhosilva@hotmail.com

${ }^{3}$ Enfermeiro. Mestre pelo Programa de Pós-Graduação Mestrado Profissional Enfermagem Assistencial (MPEA) da Escola de Enfermagem Aurora de Afonso Costa, da Universidade Federal Fluminense (EEAAC/UFF). Professor Substituto da Universidade Federal de Alagoas (UFAL). Maceió. Alagoas - Brasil. E-mail: wbiratansouza@yahoo.com.br

${ }^{4}$ Enfermeira. Doutoranda pelo Programa Acadêmico em Ciências do Cuidado em Saúde (PACCS) da Escola de Enfermagem Aurora de Afonso Costa, da Universidade Federal Fluminense (EEAAC/UFF). Mestre pelo Programa de Pós-Graduação Mestrado Profissional Enfermagem Assistencial (MPEA) da Escola de Enfermagem Aurora de Afonso Costa, da Universidade Federal Fluminense (EEAAC/UFF). Enfermeira do Instituto Nacional do Câncer (INCA). Rio de Janeiro. Rio de Janeiro - Brasil. Email: lucimere_santos@hotmail.com
\end{abstract}




\title{
Resumo
}

Devido ao crescimento e morbidade das Doenças Renais Crônicas, torna-se relevante medidas preventivas realizadas por enfermeiros, principalmente entre adolescentes. Assim, o ojetivo desse estudo é relatar o trabalho de educação em saúde desenvolvido com adolescentes, acerca das principais causas e medidas preventivas relativas à doença renal crônica. Estudo descritivo, tipo relato de experiência, realizado com 52 adolescentes numa escola técnica, acerca das principais causas e medidas preventivas relativas à doença renal crônica. O estudo foi realizado seguindo as etapas: planejamento das ações, realização de palestra, triagem de risco e entrega de material educativo impresso. Foi identificado grupo de risco para doença renal crônica, evidenciado pela presença de morbidades e hábitos que predispõem seu desenvolvimento. Observou-se carência de conhecimentos sobre doença renal, consequentemente, de medidas preventivas relativas à mesma. Ressaltou-se atuação do enfermeiro como educador em saúde, na prevenção de doenças dentro do ambiente escolar.

Palavras-chave: Nefropatias; Adolescente; Educação em enfermagem.

\begin{abstract}
Due to the growth and morbidity of Chronic Kidney Diseases, preventive measures performed by nurses, especially among adolescents, become relevant. Thus, the objective of this study is to report the health education work developed with adolescents about the main causes and preventive measures related to chronic kidney disease. A descriptive study, a type of experience report, carried out with 52 adolescents in a technical school about the main causes and preventive measures related to chronic kidney disease. The study was carried out following the steps: action planning, lecture, risk screening and delivery of printed educational material. A risk group for chronic kidney disease was identified, evidenced by the presence of morbidities and habits that predispose its development. There was a lack of knowledge about renal disease and, consequently, of preventive measures related to it. Emphasis was placed on the nurse's role as a health educator in the prevention of diseases within the school environment.
\end{abstract}

Keywords: Nephropathies; Adolescent; Nursing education. 


\section{Introdução}

A Doença Renal Crônica (DRC) é definida como perda progressiva e irreversível dos néfrons funcionais. É classificada em cinco estágios que se relacionam com a taxa de filtração glomerular e, consequentemente, com o grau de insuficiência. No estágio cinco, intitulado doença renal crônica em estágio terminal, os rins perdem o controle total da homeostasia do organismo, devido à grande redução dos néfrons ${ }^{(1)}$. Representa uma das patologias renais com aumento gradativo em proporções alarmantes, com evidências de aumento crescente na incidência e na prevalência, requerendo tratamento de alto custo do Sistema Único de Saúde (SUS) ${ }^{(2-3)}$. Surge, portanto, a necessidade da tomada de medidas de prevenção dos fatores de risco e da doença renal propriamente dita.

De acordo com as Diretrizes Brasileiras de Doença Renal Crônica, são considerados como fator de risco elevado para o desenvolvimento da doença os portadores de hipertensão arterial sistêmica, diabetes mellitus, história familiar de doença renal crônica e as doenças infecciosas ${ }^{(4)}$. Resultado da epidemia crescente dos fatores de risco cardiovasculares, a doença renal crônica implica em frequentes hospitalizações e em elevado custo socioeconômico, assumindo, nos últimos anos, o status de problema de saúde pública devido à elevação de sua prevalência entre a população mundial e ao seu impacto na morbimortalidade dos indivíduos acometidos ${ }^{(5)}$.

A Política Nacional de Atenção Integral ao Portador de Doença Renal visa prevenir a doença mediante promoção da saúde, diminuição do número de casos e minimização dos agravos da hipertensão arterial e do diabetes mellitus, que são patologias prevalentes e determinantes da doença renal na população(6).

Estima-se que existam atualmente em torno de 192 milhões de indivíduos em programa crônico de diálise segundo o censo de 2011 . Mais de $80 \%$ desses pacientes têm seu tratamento subsidiado pelo SUS. Apenas para o tratamento dessa parcela de doentes, estima-se cerca de $10 \%$ de todo o orçamento do Ministério da Saúde ${ }^{(4)}$. O crescente custo da assistência e da incorporação tecnológica em saúde, envelhecimento populacional, transição epidemiológica com aumento da incidência e prevalência das doenças crônicas, os potenciais impactos das ações para promoção da saúde, prevenção de doenças e necessidade de estimular a qualidade de vida são alguns dos fatores motivadores para busca de estratégias para o enfrentamento dos desafios postos ${ }^{(7)}$.

A adolescência, fase foco desta experiência, é um período que exige atenção especial e intervenções, representando um desafio para profissionais de saúde por ser uma fase que envolve alterações, inquietações, descobertas e desenvolvimento 
corporal, psicológico e mental. Nesta fase, com vistas à prevenção de doenças e promoção de saúde, ações de educativas adquirem grande relevância, além de ações para identificação de fatores de riscos e detecção precoce de agravos, neste caso, a doença renal crônica ${ }^{(8)}$.

Diante desses desafios, considerando também a necessidade de combate ao desenvolvimento de doenças crônicas, com intuito de favorecer a prevenção de agravos à saúde e fortalecer a relação entre as redes públicas de saúde e educação, o Ministério da Saúde criou o Programa Saúde na Escola, uma política intersetorial da Saúde e da Educação, voltadas às crianças, adolescentes, jovens e adultos da educação pública brasileira, onde ações de educação em saúde são desenvolvidas no ambiente escolar, por uma equipe multiprofissional, onde também participa o enfermeiro ${ }^{(9)}$.

Uma das atribuições do enfermeiro é a de educador. A educação em saúde se insere nas ações do enfermeiro como um processo que auxilia as pessoas a aprenderem comportamentos relacionados à saúde, que possam ser incorporados em sua vida diária, com objetivo do aprimoramento da saúde e promoção do autocuidado. O processo educativo é entendido como um curso de ação sistemático, planejado e com embasamento científico, que envolve educador e aprendiz ${ }^{(10)}$.

Nessa perspectiva, este estudo tem como objetivo relatar o trabalho de educação em saúde desenvolvido com adolescentes, acerca das principais causas e medidas preventivas relativas à doença renal crônica.

\section{Método}

Trata-se de um estudo descritivo, do tipo relato de experiência, que ocorreu em Maio de 2017, com adolescentes de uma Escola Técnica Estadual da Região dos Lagos, no estado do Rio de Janeiro, acerca das principais causas e medidas preventivas relativas à doença renal crônica. Participaram 52 alunos do segundo ano do ensino médio técnico, com faixa etária de 16 a 19 anos, selecionados a critério da direção.

A ação foi realizada por quatro enfermeiros, no contexto da disciplina de Fundamentos Teórico-Práticos do Ensino, que contribui para a iniciação a docência, ministrada no curso de Mestrado Profissional em Enfermagem Assistencial da Escola de Enfermagem Aurora de Afonso Costa, da Universidade Federal Fluminense. A autorização para realização da ação foi adquirida por meio de uma carta de anuência, enviada previamente e assinada pela direção da unidade escolar.

A atividade foi desenvolvida nas seguintes etapas:

\section{Planejamento das Ações}

Foram realizados dois encontros na unidade escolar. O primeiro encontro, com antecedência de 21 dias, teve a intenção de esclarecer sobre a ação e solicitar anuência 
para sua realização, por meio de diálogo com a direção da escola acerca do tema a ser abordado. O segundo encontro, com antecedência de 15 dias, teve o objetivo de realizar um levantamento sobre recursos existentes na escola e possibilidades de atuação. Com ajuda e sugestão do professor de biologia, devido associação entre o assunto a ser abordado e o aplicado na disciplina naquele semestre, acerca de sistema urinário, decidiu-se realizar palestra educativa, breve levantamento de fatores de risco e distribuição de material educativo impresso do tipo folder.

\section{Realização da Estratégia Educativa}

Os adolescentes das turmas selecionadas foram convidados verbalmente para o auditório, onde foram esclarecidos os propósitos do trabalho, ficando livre à participação ou não, conforme interesse de cada um. A palestra foi realizada usando-se como estratégia a apresentação de slides e vídeos com informações inerentes à anatomia e função renal, doença renal crônica e suas causas, terapias substitutivas e a relevância de medidas preventivas, considerando que a doença pode surgir em diferentes faixas etárias, inclusive na adolescência, além da evolução em longo prazo.

\section{Breve Levantamento de Fatores de Risco}

Após exposição dos principais tópicos acerca da doença renal crônica, foi realizada aferição da Pressão Arterial de todos os ouvintes, com a intenção de identificar alterações na PA dos alunos e apresentar essa prática aos mesmos, considerando a relação existente entre valores pressóricos elevados e complicações crônicas que podem se desenvolver posteriormente. A equipe de enfermeiros se dividiu e, durante a aferição da pressão arterial e também para aqueles que aguardavam sua vez de aferir, foram abordadas questões em forma de bate-papo, de modo aleatório e informal, a saber: Você é hipertenso ou diabético?; Tem alguém diabético ou hipertenso na família?; Há alguém com doença renal na família?; Consume alimentos com muito sal?; Já participou de alguma palestra sobre prevenção de doença renal crônica?; Já fez exame para verificação de glicemia?. Para os que aguardavam solicitou-se que levantassem as mãos, em caso de resposta positiva.

\section{Entrega de Material Educativo Impresso}

No sentido de favorecer o aprendizado e a disseminação das informações, a atividade foi concluída com entrega de folder educativo com os principais tópicos acerca da doença renal crônica. Para potencializar a ação e avaliar o conhecimento adquirido pelos adolescentes durante as ações educativas foi realizada a leitura do folder e uma breve discussão sobre os assuntos contidos no material, com objetivo de sanar possíveis dúvidas e reforçar as informações. Desse modo, as dúvidas que surgiram foram esclarecidas e os alunos verbalizaram os 
conhecimentos adquiridos acerca do tema. Utilizando a oportunidade do momento, solicitou-se que os mesmos dividissem as informações obtidas com amigos e familiares, enfatizando a importância da atuação como multiplicadores das informações.

Em todas as etapas do estudo, os termos técnicos inerentes à área da saúde foram substituídos por termos mais simples, populares e didáticos, a fim de atrair a atenção dos adolescentes, bem como promover melhor compreensão sobre o tema abordado.

\section{Relato de Experiência}

Participaram da ação 52 adolescentes, com faixa etária de 16 a 19 anos. Com relação ao gênero, foram 38 do sexo feminino e 14 do sexo masculino. Dos 52 participantes, quando perguntados se já haviam participado de alguma palestra sobre a prevenção da doença renal crônica, 40 deles responderam que não e apenas 12 responderam sim, tornando evidente a deficiência sobre a abordagem desse tema dentro da referida faixa etária. Houve boa aceitação da temática e todos participaram das diferentes etapas.

Alguns adolescentes apresentaram suscetibilidade aumentada para doença renal crônica, por meio do breve levantamento anteriormente descrito, e foram considerados grupo de risco, conforme resposta positiva para os questionamentos durante aferição da pressão arterial, considerando que: A hipertensão arterial é comum na doença renal crônica, podendo ocorrer em mais de $75 \%$ dos pacientes de qualquer idade. Os diabéticos apresentam risco aumentado para doença renal crônica e doença cardiovascular, e devem ser monitorizados frequentemente quanto à ocorrência da lesão renal; Os familiares de portadores de doença renal crônica apresentam prevalência aumentada de hipertensão arterial, diabetes mellitus, proteinúria e doença renal ${ }^{(11)}$.

Foi possível perceber o interesse dos participantes em relação à obtenção de informações inerentes aos cuidados com a saúde. Alguns alunos afirmaram que se cuidavam, mas muitos deles sinalizaram que exageram no consumo de alimentos ricos em sal. Ao serem indagados a respeito da aferição da pressão arterial e verificação da glicemia capilar, a maioria dos alunos já haviam aferido a pressão arterial e poucos tinham conhecimento acerca da glicemia capilar.

A doença renal crônica associa-se com maior risco de morbimortalidade e determina um grande impacto financeiro no sistema de saúde. Os altos custos estão relacionados com consulta médica, medicações e exames necessários para detecção da doença, além dos gastos com terapias substitutivas ${ }^{(12)}$. Porém, é uma doença prevenível e evitável, e pode ser detectada através de exames simples e baratos disponibilizados pelo SUS.

Atualmente, poucos são os levantamentos epidemiológicos voltados para doença renal crônica em crianças e adolescentes, qualquer que seja o estágio da 
evolução da doença. Aumentar ações educativas e de conscientização sobre doenças renais e doença renal na infância e adolescência, são atividades harmoniosas com os objetivos da Organização Mundial da Saúde para reduzir a mortalidade por doenças não transmissíveis, com foco em mudanças no estilo de vida, incluindo a redução do consumo de tabaco, controle de consumo de sal, controle de energia da dieta e redução de ingestão de álcool, e intervenções efetivas incluindo pressão arterial, colesterol e controle glicêmico(3).

Evidenciada a importância da promoção à saúde de adolescentes e jovens, enfatiza-se a necessidade de estabelecer processos de intervenção intersetoriais e interdisciplinares, de mudanças na gestão e trabalho das equipes de saúde para interligar esses dois campos da atenção à saúde, e as escolas concentram grandes números de adolescentes e jovens, sendo um local favorável para projetos de prevenção de doenças ${ }^{(13)}$.

Neste sentido, destaca-se o papel do enfermeiro como educador em diferentes cenários, sobretudo no ambiente escolar. No tocante à organização da atenção ao adolescente na Atenção Primária de Saúde, as políticas definem a escola como contexto situacional para as práticas de cuidado a esse grupo social, compreendendo a escola como um espaço privilegiado para construção de valores pessoais, de crenças, de conceitos e maneiras de conhecer o mundo ${ }^{(14)}$.
Compreender diagnósticos de alto risco e eventos que ocorrem na infância tem o potencial para identificar e intervir preventivamente nas pessoas com o maior risco de desenvolver doença renal crônica durante suas vidas. Concentrando-se na doença renal na infância, soluções de baixo custo podem ser realizadas, como o tratamento precoce e medidas preventivas da doença podem impedir a instalação tardia da doença renal avançada ${ }^{(15)}$. Considerando as principais causas da doença renal crônica de origem hereditária como a diabetes e hipertensão, tornam-se relevantes medidas de prevenção em todas as idades.

Enquanto a Estratégia Saúde da Família tem como centralidade ações de promoção da saúde e de prevenção e controle das doenças centradas na família, o Programa Saúde na Escola tem como finalidade contribuir para formação integral dos estudantes da rede pública de Educação Básica por meio de ações de prevenção, promoção e atenção à saúde. Dentre os objetivos do programa de saúde na escola destaca-se a articulação das ações de saúde e educação, de forma a ampliar o alcance e impacto das ações e enfrentamento das vulnerabilidades ${ }^{(14)}$.

Vale ressaltar que as doenças renais podem ser prevenidas se detectadas precocemente, dessa forma o conhecimento dos sinais e sintomas pode se fazer necessário para evitar a evolução da doença. São necessários maiores esforços para realinhar e expandir essas colaborações 
multidisciplinares, com foco mais agudo na detecção e tratamento precoces da doença renal ${ }^{(3)}$.

As ações de enfermagem no âmbito escolar, voltadas para a promoção da saúde, devem ser centralizadas no ser humano dentro de uma perspectiva integral. O ambiente escolar, por ser diariamente frequentado pelo adolescente, é propício ao desenvolvimento de estratégias educativas de prevenção, uma vez que a educação em saúde promove reflexão acerca do tema abordado, implicando em mudança de comportamento $^{(15)}$.

A adolescência pode ser uma fase de risco, devido aos hábitos pouco saudáveis assumidos por muitos adolescentes, portanto obter conhecimento a respeito do binômio saúde/doença proporciona o empoderamento do indivíduo quanto ao autocuidado, sendo assim, destaca-se a incumbência mais nobre do enfermeiro que se refere à educação em saúde.

\section{Conclusão}

Essa atuação contribuiu para melhoria da qualidade de vida de jovens e adolescentes, através de educação em saúde sobre a doença renal crônica e destacou a relevância da prevenção dessa doença. Vale ressaltar a atuação do enfermeiro como agente educador e que a assistência de enfermagem transcende as fronteiras hospitalares e de unidades de saúde, pois as medidas educativas e preventivas podem ocorrer em diferentes lugares, sendo a escola um cenário bem-sucedido para propagar informações sobre prevenção de doenças.

Notou-se a necessidade da adoção de medidas preventivas da doença renal entre adolescentes e que além de projetos educativos realizados na escola, outras iniciativas de informações e prevenção de doenças devem ser tomadas, principalmente utilizando mídias de grande alcance de massas. A linguagem utilizada durante a educação em saúde para adolescentes é fator determinante para o sucesso do processo educativo. $\mathrm{O}$ enfermeiro assume papel fundamental como educador, para proporcionar informações relativas á prevenção de doenças, fomentando o empoderamento do cuidado com a saúde entre adolescentes.

\section{Referências}

1. Tavares JMAB, Lisboa MTL. Tratamento com diálise peritoneal: a prática do autocuidado no contexto familiar. Rev enferm UERJ [Internet]. 2015; 23(3): 344-9. Disponível em http://www.facenf.uerj.br/v23n3/v23n3a09.p df.

2. Melo WF, Bezerra ALD, Sousa MNA. Perfil epidemiológico de pacientes com insuficiência renal crônica: um estudo quantitativo. C\&DRevista Eletrônica da Fainor [Internet]. 2014; 7(2): 142-156. Disponivel em: http://srv02.fainor.com.br/revista/index.php/ $\mathrm{m} 02$.

3. Ingelfinger JR, Kalantar-Zadeh K, Schaefer F. Em tempo: evitando as consequências da doença renal - foco na infância. Rev Paul Pediatr [Internet]. 2016; 34(1): 5-10. Disponível

em: 
http://www.scielo.br/pdf/rpp/v34n1/pt_0103 -0582-rpp-34-01-0005.pdf.

4. Cruz CF, Cunha GOD, Souza SRP. Custo do tratamento dos pacientes com insuficiência renal crônica em estágio terminal no município de São Paulo, no período de 2008 a 2012. Science in Health [Internet]. 2014; 5(1): 6-11. Disponível em: http://arquivos.cruzeirodosuleducacional.edu. $\mathrm{br} /$ principal/new/revista_scienceinhealth/13 j an_abr_2014/Science_05_01_6-11.pdf.

5. Pinho NA, Silva GV, Pierin AMG. Prevalência e fatores associados à doença renal crônica em pacientes internados em um hospital universitário na cidade de São Paulo, SP, Brasil. J BrasNefrol [Internet]. 2015; 37(1): 9197. Disponível em: http://www.scielo.br/pdf/jbn/v37n1/01012800-jbn-37-01-0091.pdf.

6. Ministério da Saúde (BR). Portaria GM/MS 1.168 , de 15 de junho de 2004. Institui a política nacional de atenção ao Portador de Doença Renal. Diário Oficial da União, 2004. Disponível em: http://bvsms.saude.gov.br/bvs/publicacoes/p ortaria_1168_ac.htm.

7. Ministério da Saúde (BR). Agência Nacional de Saúde Suplementar. Cartilha para a modelagem de programas para promoção da saúde e prevenção de riscos e doenças. Rio de Janeiro: ANS; 2011. 80 p. Disponível em: http://www.ans.gov.br/images/stories/Materi ais_para_pesquisa/Mat_web.pdf.

8. Ribeiro VCS, Nogueira DL, Assunção RS, Resende e Silva FM, Quadros KAN. Papel do enfermeiro da estratégia de saúde da família na prevenção da gravidez na adolescência. $R$. Enferm. Cent. O. Min. 2016; 1(6):1957-1975. Disponível em: http://seer.ufsj.edu.br/index.php/recom/articl e/view/881/1006.

9. Ministério da Educação (BR). Plano Nacional de Educação. Programa Saúde na Escola (PSE): intersetorialidade como premissa para desenvolvimento integral. Brasília: Observatório do PNE; 2015. Disponível em: http://educacaointegral.org.br/experiencias/p r/.

10. Arruda LP, Moreira ACA, Aragão AEA. Promoção da saúde: atribuições do enfermeiro como educador na estratégia saúde da família. Essentia [Internet]. 2014; 16(1): 183-203. Disponível em: file:///C:/Users/Particular/Downloads/10-401-PB.pdf.

11. National Kidney Foundation K/DOQI. clinical practice guidelines for chronic kidney disease: evaluation, classification and stratification. Am J KidneyDis. [Internet]. 2002; 39(Supl2):1-246. Disponível em: https://www.kidney.org/sites/default/files/do cs/ckd_.pdf.

12. Ferreira CDM. Análise de custos da doença renal crônica nos estágios 3 a 5 pré- dialítico para o Sistema Único de Saúde[Dissertação]. Juiz de Fora: Universidade Federal de Juiz de Fora; 2012. Disponível em: http://www.ufjf.br/pgsaudecoletiva/files/201 3/03/AN\%C3\%81LISE-DE-CUSTOS-CD.pdf.

13. Ministério da Saúde (BR). Secretaria de Atenção em Saúde. Departamento de Ações Programáticas Estratégicas. Diretrizes nacionais para a atenção integral à saúde de adolescentes e jovens na promoção, proteção e recuperação da saúde. / Ministério da Saúde, Secretaria de Atenção em Saúde, Departamento de Ações Programáticas Estratégicas, Área Técnica de Saúde do Adolescente e do Jovem. Brasília: Ministério da Saúde; 2010. Disponível em: http://bvsms.saude.gov.br/bvs/publicacoes/di retrizes_nacionais_atencao_saude_adolescent es_jovens_promocao_saude.pdf.

14. Nogueira ALA, Sousa FGM, Silva IR, Silva ACO, DCM Silva, EEC Santana. Funcionalidade de famílias de adolescentes de escolas públicas: uma abordagem descritiva. Cogitare Enferm. [Internet] 2012; 17(2):224-31. Disponível em: http://www.redalyc.org/articulo.oa?id=48364 8963003. 
15. Pedrosa SC, Costa DVS, Citó MCO, Luna IT, Pinheiro PNC. Educação em saúde com adolescentes acerca do uso de álcool e outras drogas.R. Enferm. Cent. O. Min. 2015; 5(1): 1535-1541. Disponível em: http://seer.ufsj.edu.br/index.php/recom/articl e/view/402/843. 